\title{
Superlattice approach to doping infinite-layer nickelates
}

\author{
R. A. Ortiz $\odot,{ }^{1}$ H. Menke, ${ }^{2}$ F. Misják,${ }^{3}$ D. T. Mantadakis, ${ }^{1}$ K. Fürsich $\odot,{ }^{1}$ E. Schierle, ${ }^{4}$ G. Logvenov, \\ U. Kaiser, ${ }^{3}$ B. Keimer, ${ }^{1}$ P. Hansmann ${ }^{1},{ }^{2,5},{ }^{*}$ and E. Benckiser ${ }^{1, \dagger}$ \\ ${ }^{1}$ Max Planck Institute for Solid State Research and Center of Integrated Quantum Network, Heisenbergstraße 1, 70569 Stuttgart, Germany \\ ${ }^{2}$ Department of Physics, University of Erlangen-Nürnberg, 91058 Erlangen, Germany \\ ${ }^{3}$ Central Facility of Electron Microscopy, Ulm University, Albert-Einstein-Allee 11, 89081 Ulm, Germany \\ ${ }^{4}$ Helmholtz-Zentrum Berlin für Materialien und Energie, Albert-Einstein-Straße 15, 12489 Berlin, Germany \\ ${ }^{5}$ Max Planck Institute for Chemical Physics of Solids, Nöthnitzerstraße 40, 01187 Dresden, Germany
}

(Received 29 May 2021; accepted 4 October 2021; published 22 October 2021)

\begin{abstract}
The recent observation of superconductivity in infinite-layer $\mathrm{Nd}_{1-x} \mathrm{Sr}_{x} \mathrm{NiO}_{2}$ thin films has attracted a lot of attention, since this compound is electronically and structurally analogous to the superconducting cuprates. Due to the challenges in the phase stabilization upon chemical doping with $\mathrm{Sr}$, we synthesized artificial superlattices of $\mathrm{LaNiO}_{3}$ embedded in insulating $\mathrm{LaGaO}_{3}$, and we used layer-selective topotactic reactions to reduce the nickelate layers to $\mathrm{LaNiO}_{2}$. Hole doping is achieved via interfacial oxygen atoms and tuned via the layer thickness. We used electrical transport measurements, transmission electron microscopy, and x-ray spectroscopy together with $a b$ initio calculations to track changes in the local nickel electronic configuration upon reduction, and we found that these changes are reversible. Our experimental and theoretical data indicate that the doped holes are trapped at the interfacial quadratic pyramidal Ni sites. Calculations for electron-doped cases predict a different behavior, with evenly distributed electrons among the layers, thus opening up interesting perspectives for interfacial doping of transition-metal oxides.
\end{abstract}

DOI: 10.1103/PhysRevB.104.165137

\section{INTRODUCTION}

Ever since the discovery of unconventional superconductivity in high- $T_{\mathrm{c}}$ cuprates, the search has been ongoing for other $3 d$ transition-metal oxides that exhibit this intriguing quantum state of matter. This is especially true for nickelates, because nickel is right next to copper in the Periodic Table. Recently, the first observation of superconductivity in heteroepitaxially grown thin films of $\mathrm{Sr}$-doped $\mathrm{NdNiO}_{2}$ raised a lot of interest [1]. Some time passed before these results could be reproduced experimentally, which is largely related to the delicate growth conditions [2]. Similarly to the high- $T_{\mathrm{c}}$ cuprates, the phase diagram of infinite-layer nickelates shows a domelike doping dependence for the superconducting transition temperature $T_{\mathrm{c}}$ [2-4]. The optimal doping with maximum $T_{\mathrm{c}} \approx 15 \mathrm{~K}$ has been reported for $\mathrm{Nd}_{0.8} \mathrm{Sr}_{0.2} \mathrm{NiO}_{2}$. The synthesis route of this compound requires the growth of a precursor $\mathrm{Nd}_{0.8} \mathrm{Sr}_{0.2} \mathrm{NiO}_{3}$ thin film, which is subsequently treated by a soft-chemistry reduction with $\mathrm{CaH}_{2}$. The preparation of the precursor film constitutes the first challenge, since the $\mathrm{Sr}$ doped perovskite competes with Ruddlesden-Popper phases,

\footnotetext{
*philipp.hansmann@fau.de

†E.Benckiser@fkf.mpg.de
}

Published by the American Physical Society under the terms of the Creative Commons Attribution 4.0 International license. Further distribution of this work must maintain attribution to the author(s) and the published article's title, journal citation, and DOI. Open access publication funded by the Max Planck Society. leaving a very narrow growth window [5]. The homogeneity of the Sr-dopant distribution and oxygen reduction, as well as the role of the heteroepitaxy with $\mathrm{SrTiO}_{3}$, impose further challenges for the synthesis, with many details that remain yet to be explored.

The soft-chemistry reduction of nickelate powders and thin films was studied for the first time several years ago [6-12]. Starting from $\mathrm{LaNiO}_{3}$, the reduction to $\mathrm{LaNiO}_{2}$ takes place via the formation of relatively stable phases with intermediate oxygen content, $\mathrm{LaNiO}_{2.75}$ and $\mathrm{LaNiO}_{2.5}$ [13]. Structurally, the reduction goes along with successive removal of apical oxygen ions, such that for the so-called brownmillerite phase $\mathrm{LaNiO}_{2.5}$, columns of $\mathrm{Ni}$ ions with square-planar coordination are placed between the octahedrally coordinated nickel ions in the perovskite phase. The lattice parameters change from nearly cubic $3.84 \AA$ for $\mathrm{LaNiO}_{3}$ to enlarged in-plane $a=$ $3.96 \AA$ (perpendicular to the $\mathrm{NiO}_{4}$ columns) and reduced outof-plane $c=3.38 \AA$ values in $\mathrm{LaNiO}_{2}[6,10]$. The collapse of the $c$-axis parameter makes the reduction process trackable by x-ray diffraction (XRD) and high-resolution transmission electron microscopy (HRTEM), even for very thin films and multilayers.

Theoretical interest in this new, supposedly unconventional superconductor naturally arises from the possible similarity to, or insightful differences from, high- $T_{\mathrm{c}}$ cuprates [14-18]. Some theories predict much weaker magnetic correlations for the infinite-layer nickelates, and thus question their relevance for the superconducting pairing mechanism [19]. Other theoretical studies, however, come to different conclusions [18] in agreement with recent results from paramagnon dispersion 


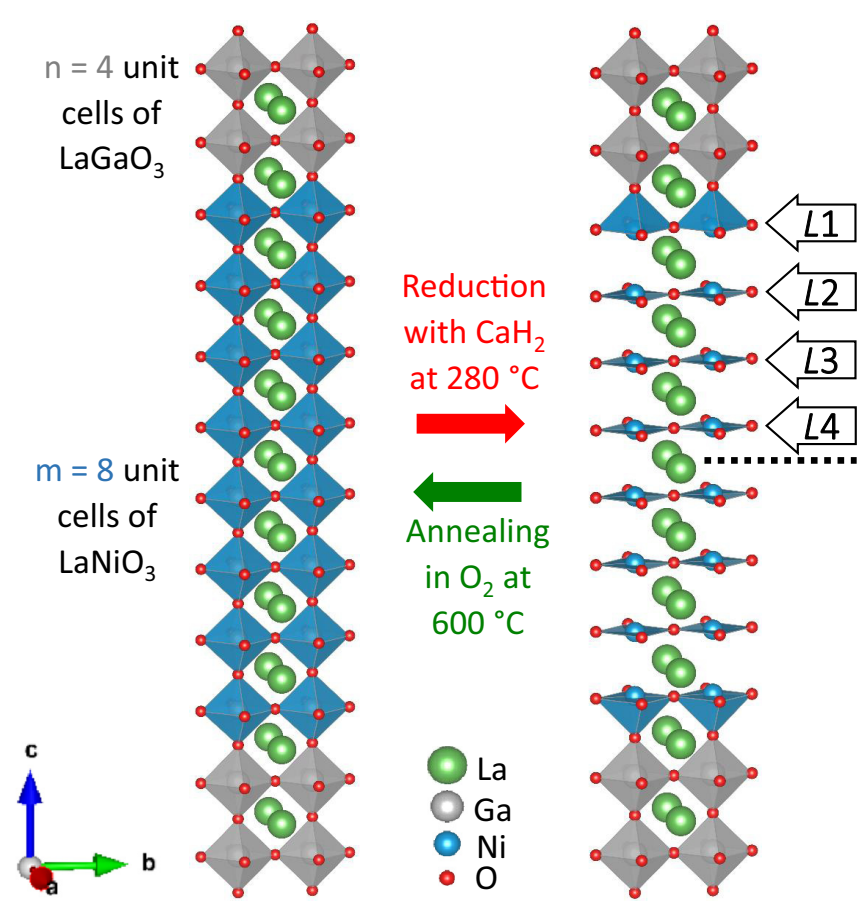

FIG. 1. Left: Sketch of the pristine (reoxidized) $\mathrm{LaNiO}_{3}$ (blue)$\mathrm{LaGaO}_{3}$ (gray) superlattice with $m=8, n=4$ stacking sequences grown on a (001) $\mathrm{SrTiO}_{3}$ substrate. Right: Structure stabilized by layer-selective reduction of the nickelate slab with $\mathrm{CaH}_{2}$. We label individual layers within the nickelate slab by $L 1-L 4$. The dashed horizontal line indicates the mirror plane.

analysis $[20,21]$. Furthermore, $\mathrm{Ni}^{1+}$ with a formal $3 d^{9}$ valence electron configuration is rarely found in bulk compounds, and the hybridization strength with the oxygen ligands appears to be weaker than the one of $\mathrm{Cu}^{2+}$ in cuprates [22,23].

Here we report on an alternative approach to holedoping infinite-layer nickelates that takes advantage of the layer-selective topotactical reduction [24] of $\left(\mathrm{LaNiO}_{3}\right)_{m} /$ $\left(\mathrm{LaGaO}_{3}\right)_{n}$ superlattices grown on $(001) \mathrm{SrTiO}_{3}$ substrates. The essential idea is that in a layer-selective reduction process, the interfacial, apical oxygen ions remain, forming a structure depicted in Fig. 1. A similar interface structure has also been suggested in recent density functional theory studies $[15,25]$. Assuming that the additional charge originating from the interfacial oxygen ions is homogeneously distributed in the $\mathrm{LaNiO}_{2}$ layer stack, this opens the possibility of a tunable doping level, where the average nickel valence state $\mathrm{Ni}^{1+\frac{2}{m}}$ is controlled by the number of consecutive layers $m$ (see Fig. 1). A similar doping mechanism is assumed to occur in reduced Ruddlesden-Popper (RP) single crystals of $(\mathrm{La}, \mathrm{Pr})_{4} \mathrm{Ni}_{3} \mathrm{O}_{8}$ [26]. A recent report shows that optimally self-doped, epitaxial RP thin films exhibit superconductivity [27]. Another advantage of this approach is that the growth of the precursor superlattices is stable and the optimal conditions have been well established [28].

\section{SYNTHESIS AND CHARACTERIZATION OF SUPERLATTICES}

In the following, we focus on $\left(\mathrm{LaNiO}_{3}\right)_{m=8} /\left(\mathrm{LaGaO}_{3}\right)_{n=4}$ superlattices grown on (001) $\mathrm{SrTiO}_{3}$ substrates (see Fig. 1).
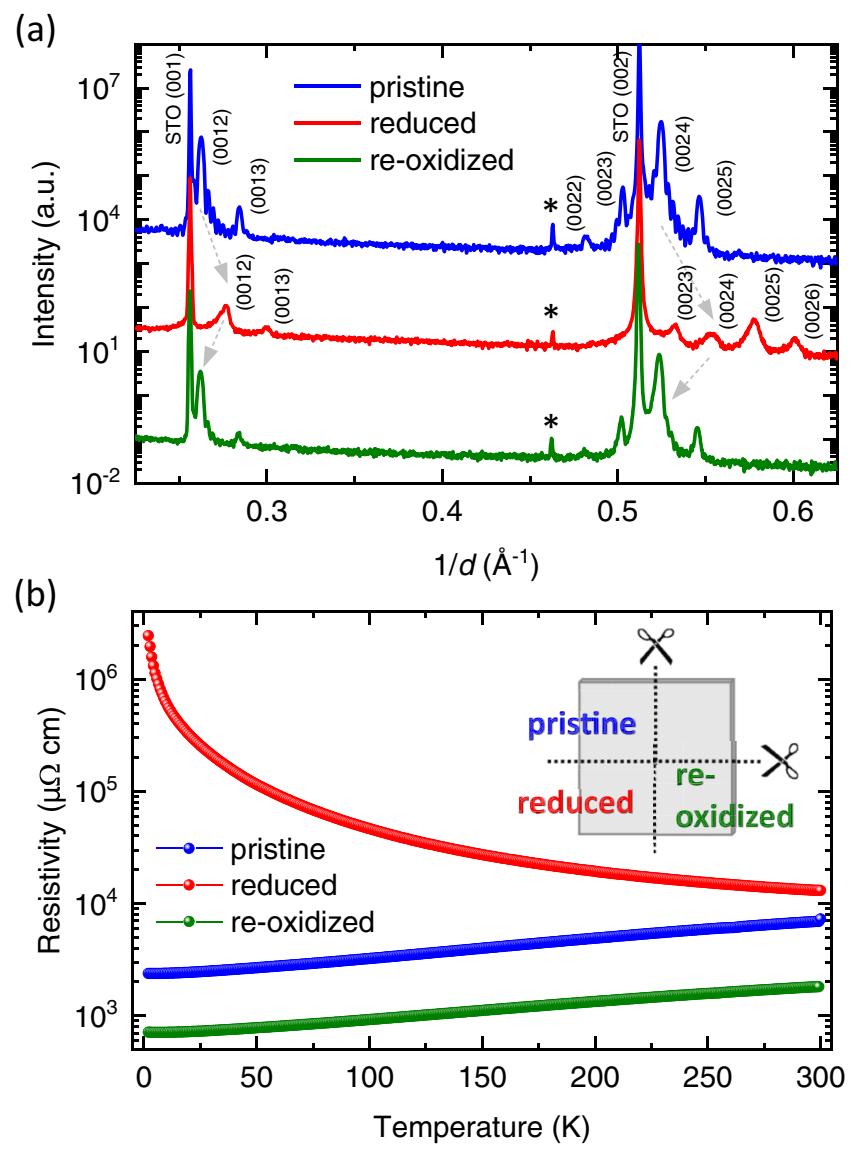

FIG. 2. (a) XRD patterns of a set of pristine, reduced, and reoxidized $\mathrm{LaNiO}_{3(2+\delta)} / \mathrm{LaGaO}_{3}$ superlattices including the (001) and (002) reflections of the $\mathrm{SrTiO}_{3}$ substrate. The asterisks mark peaks arising from the $K_{\beta 1}$ reflection of the main substrate peak. The data in panel (a) have been translated vertically for clarity. (b) Resistivity of the same set of samples.

With respect to the bulk perovskite nickelate, the substrate induces a moderate tensile strain that translates into an enlarged $c$ axis parameter for the epitaxially strained infinite-layer phase. $\mathrm{LaGaO}_{3}$ was chosen to facilitate layer-selective reduction, since it is expected to be chemically more stable against oxygen removal (the $\mathrm{Ga}^{3+}$ ions have a closed-shell configuration). Further, there is no change of the La cation sublattice across the interfaces (except from the out-of-plane La atomic distances), which removes a possible source of interfacial disorder. We emphasize that superconductivity was found in analogous infinite-layer cuprate superlattices [29].

The superlattices were grown by pulsed-laser deposition (PLD) using high-density stoichiometric targets of $\mathrm{LaNiO}_{3}$ and $\mathrm{LaGaO}_{3}$ under the conditions reported in Ref. [28]. To reduce the influence of small differences in superlattice structures, samples cut out of a single growth were either kept pristine or reduced. Some of the reduced samples were subsequently reoxidized [inset in Fig. 2(b)]. The as-grown superlattices were reduced using $\mathrm{CaH}_{2}$ powder as a reduction agent. Specimens with dimensions $2.5 \times 2.5 \mathrm{~mm}^{2}$ were placed under an Ar atmosphere inside an open aluminum foil box on top of approximately $50 \mathrm{mg} \mathrm{CaH}_{2}$ powder. The quartz tubes were then sealed in vacuum $\left(10^{-6}-10^{-7}\right.$ mbar $)$ 
and subsequently annealed at $280^{\circ} \mathrm{C}$ for $72 \mathrm{~h}$. X-ray diffraction measurements $(\theta / 2 \theta$ scans) were carried out with a custom-made four-circle single-crystal diffractometer, which is equipped with a $\mathrm{Cu} K \alpha 1$ source and a Dectris MYTHEN line detector. Both $\mathrm{x}$-ray diffraction and electrical transport measurements (performed in van-der-Pauw geometry) show that the reduction process is reversible [Figs. 2(a) and 2(b)]. Such reversibility of a topotactic reduction has been previously reported in $\mathrm{LaNiO}_{2}$ thin films [11] and $\mathrm{CaFeO}_{2}$-based superlattices [24]. HRTEM images show well-ordered superlattice structures with sharp interfaces (see Appendix A) and confirm the layer selectivity of the reduction process. The collapse of the $c$-axis parameter only occurs in the $\mathrm{LaNiO}_{2+\delta}$ layers. Similar lattice parameter contraction of reduced $\mathrm{Nd}_{1-x} \mathrm{Sr}_{x} \mathrm{NiO}_{2}$ thin films was observed by annular bright-field scanning-TEM, close to the substrate [5]. The averaged bilayer thicknesses of 46.2(2) and 44.0(6) A for pristine and reduced samples, respectively, are in good agreement with the corresponding XRD values of 45.7(1) and 43.4(1) A.

While the electrical transport of pristine and reoxidized samples exhibits metallic behavior closely analogous to bulk $\mathrm{LaNiO}_{3}$, the resistivity of the reduced superlattices shows a semiconducting temperature dependence [Fig. 2(b)]. We found that the conductivity of the reoxidized samples is reproducibly higher than that of the pristine ones. We attribute this finding to the presence of oxygen vacancies in the as-grown samples. Although samples were annealed in a 1-bar oxygen atmosphere at $690^{\circ} \mathrm{C}$ as the last step of the PLD growth procedure, this annealing of the pristine samples is less efficient than reduction and subsequent annealing. While the filling of individual, isolated oxygen vacancies in the annealing process of pristine samples is limited by oxygen diffusion, this restriction is lifted when the apical oxygen sites are first emptied and then refilled.

Detailed analysis of the $(00 L)$ x-ray diffraction scans and comparison with patterns calculated by the simulation software QUAD [30] [Figs. 3(a) and 3(b)] confirmed the interfacial structure depicted in Fig. 1. In particular, the relative intensities of the $L=23,24,25$ superlattice reflections (highlow-high) are in good agreement with the simulation of quadratic pyramidal Ni-O coordination, while the monotonically increasing intensity pattern predicted for square-planar coordination disagrees with the experimental data. This characteristic intensity pattern was observed in various samples after topotactic reduction.

\section{X-RAY ABSORPTION SPECTROSCOPY}

To study modifications of the electronic configuration of the different ions upon reduction, we examined soft x-ray absorption spectra (XAS) across several relevant absorption edges at room temperature. The measurements were carried out at the UE46 PGM-1 undulator beamline of the BESSYII synchrotron at Helmholtz-Zentrum Berlin, which supplies fully linear $\sigma$ - and $\pi$-polarized light. We measured simultaneously in total-electron yield mode and fluorescence yield mode (the information on which data are shown is provided in the respective figure captions). X-ray linear dichroism was measured at a fixed incident angle of $45^{\circ}$ by switching the polarization. In this geometry, the $\sigma$ polarization corresponds to

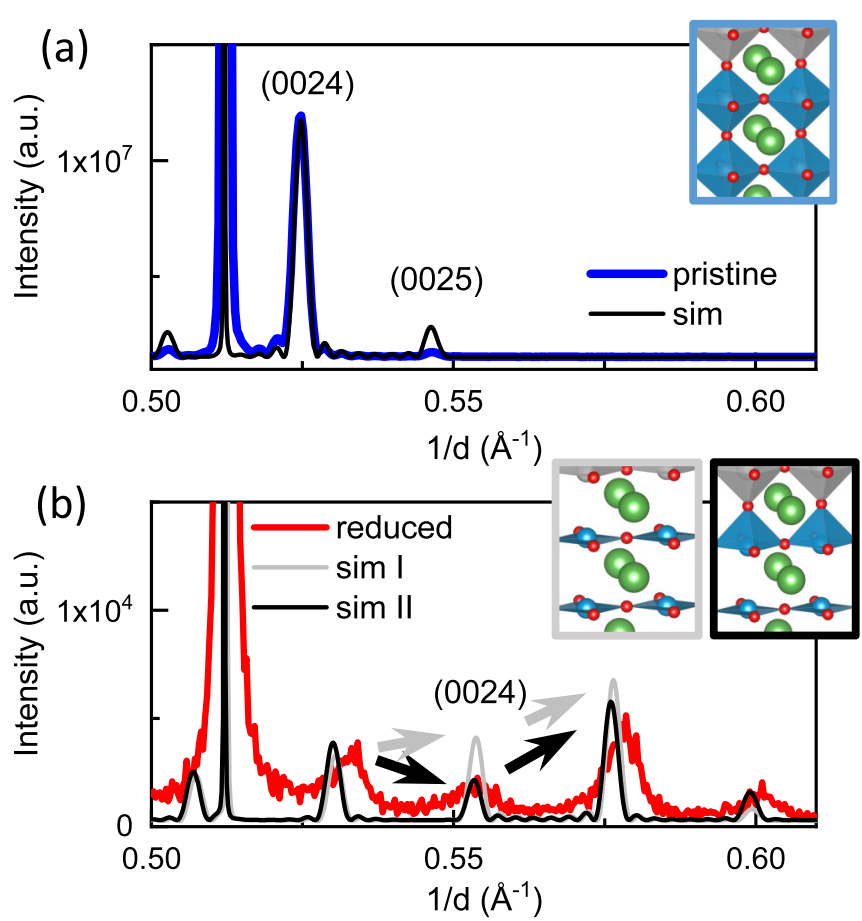

FIG. 3. QUAD simulations of the XRD data around the (002) $\mathrm{SrTiO}_{3}$ substrate reflections for different crystal structures of the (a) pristine $\mathrm{LaNiO}_{3}$ and (b) reduced $\mathrm{LaNiO}_{2+\delta}$ superlattices. In panel (b), the simulation of the proposed quadratic pyramidal (black box inset) interfacial nickel coordination yields a better agreement with the experimental results than the square planar (gray box inset).

$E \| c$ as measured; the spectra for $E \perp c$ have been obtained from the intensity $I_{\pi}$ measured with $\pi$ polarization by $I_{E \|_{c}}=$ $2 I_{\pi}-I_{E \perp c}$, with $I_{E \perp c}=I_{\sigma}$ being the intensity measured with $\sigma$ polarization [31]. Polarization-averaged spectra, calculated using $I_{\text {average }}=\left(2 I_{E \perp c}+I_{E \| c}\right) / 3$, around the La $M_{5,4}(3 d \rightarrow$ $4 f)$ and $\mathrm{Ni} L_{3,2}(2 p \rightarrow 3 d)$ absorption edges, are shown in Fig. 4(a). Compared to the pristine piece of our sample, the center of mass of the $\mathrm{Ni} L$ white lines of the reduced sample is shifted to lower energies by about $1.5 \mathrm{eV}$, which indicates a reduced $\mathrm{Ni}$ valence state. On the contrary, the La $M$ and $\mathrm{Ga} L$ lines remain unchanged within in our energy resolution. The absence of a shift and the nearly identical line shapes of the $\mathrm{Ga} L$ edge spectra before and after reduction are strong indications of the layer selectivity of the reduction [Fig. 4(b)]. The comparison of spectra at the $\mathrm{O} K$ edge, on the other hand, indicates a strongly modified Ni-O hybridization [Fig. 4(c)]. Perovskite $\mathrm{LaNiO}_{3}$ is a negative charge-transfer material, where the largest contribution to the $\mathrm{Ni}$ groundstate configuration is $3 d^{8} \underline{L}$ (where $\underline{L}$ denotes a ligand hole) [32]. This strong hybridization with nearly one hole in the oxygen $2 p$ states results in a pronounced $\mathrm{O} K$ edge prepeak around $528 \mathrm{eV}$. In the spectrum of the reduced superlattice, the spectral weight shifts towards higher energies and decreases considerably, indicating a loss of hybridization. A related effect has been observed in resonant inelastic x-ray scattering experiments on $\mathrm{NdNiO}_{2}$ and in model calculations predicting that the infinite-layer nickelates fall into the Mott-Hubbard regime, where the lowest-energy electron-addition states are 
(a)
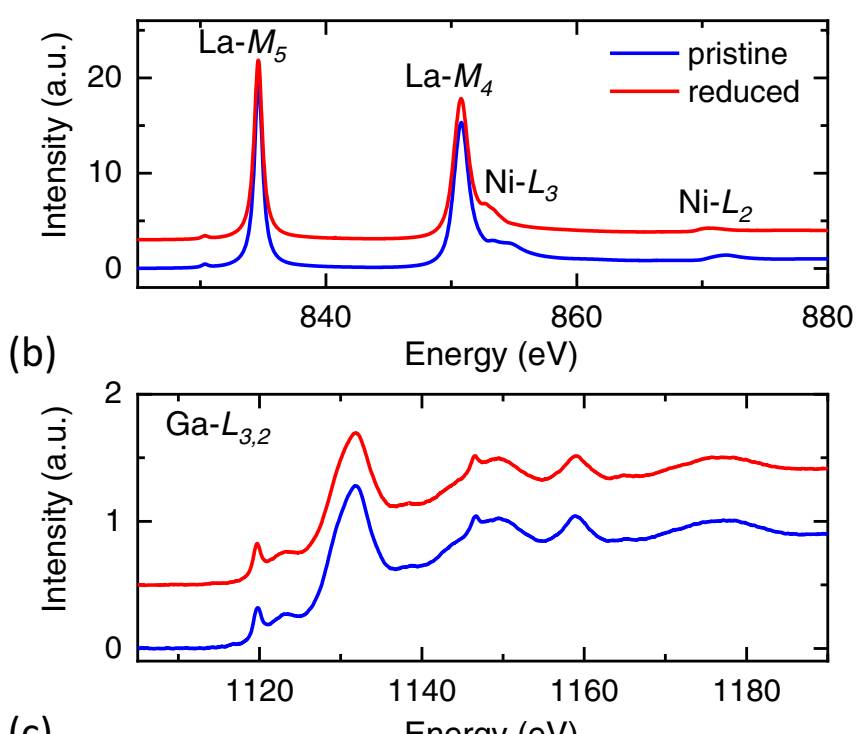

(c)

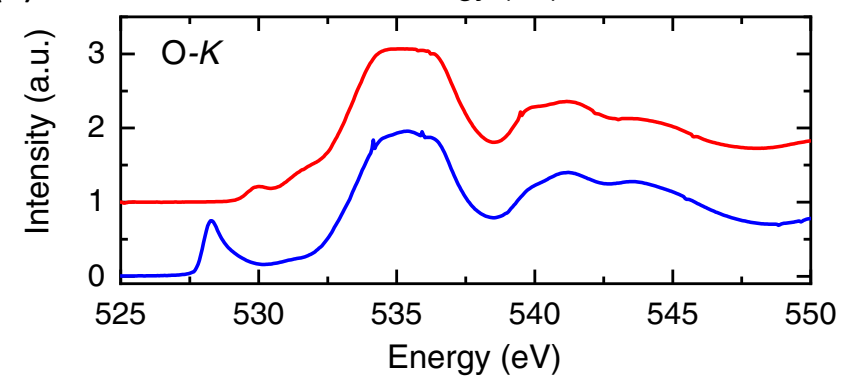

FIG. 4. Polarization-averaged XAS spectra $\left[I_{\text {average }}=\left(2 I_{E \perp c}+\right.\right.$ $\left.I_{E \|_{c}}\right) / 3$; see Fig. 5] of the energy range covering (a) La $M_{5,4}$ and $\mathrm{Ni} L_{3,2}$ edges, (b) of the $\mathrm{Ga} L_{3,2}$ edges (smoothed because of the lower flux at these high energies), and (c) around the $\mathrm{O} K$ edge. In each panel, data for pristine and reduced samples are offset for clarity. All spectra were measured in total-electron yield detection mode.

of Ni $3 d$ character $[23,33]$. Such a two-dimensional, narrowband electron system is prone to localization, in agreement with the electronic transport data of Fig. 2(b).

Next we turn to the polarization dependence of the XAS spectra. The spectra of pristine $\mathrm{LaNiO}_{3}$ are nearly isotropic, because the two $d$-shell holes on the octahedrally coordinated $\mathrm{Ni}^{3+}$ ions are approximately evenly distributed over the

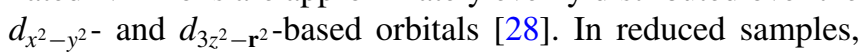
on the other hand, a strong difference between XAS spectra with x-rays polarized along $\left(I_{E \| c}\right)$ and perpendicular to the $c$-axis $\left(I_{E \perp c}\right)$ is expected to arise from the single hole on the $\mathrm{Ni}^{1+}$ ions in planar coordination, which favors the $d_{x^{2}-y^{2}}$ orbital (inset in Fig. 5). In line with this expectation, we only observed a weak negative dichroism (defined as $I_{E \perp c}-I_{E \| c}$ ) in the pristine samples (blue lines in Fig. 5), which can be attributed to combined effects of epitaxial tensile strain from the $\mathrm{SrTiO}_{3}$ substrate and electronic confinement by the insulating $\mathrm{LaGaO}_{3}$ spacer layers [28]. As expected from the change in electron filling and crystal field, the dichroism of the reduced sample is greatly enhanced and shows a change in sign. Its line shape is remarkably similar to the dichroism of $\mathrm{Cu}^{2+}$ in

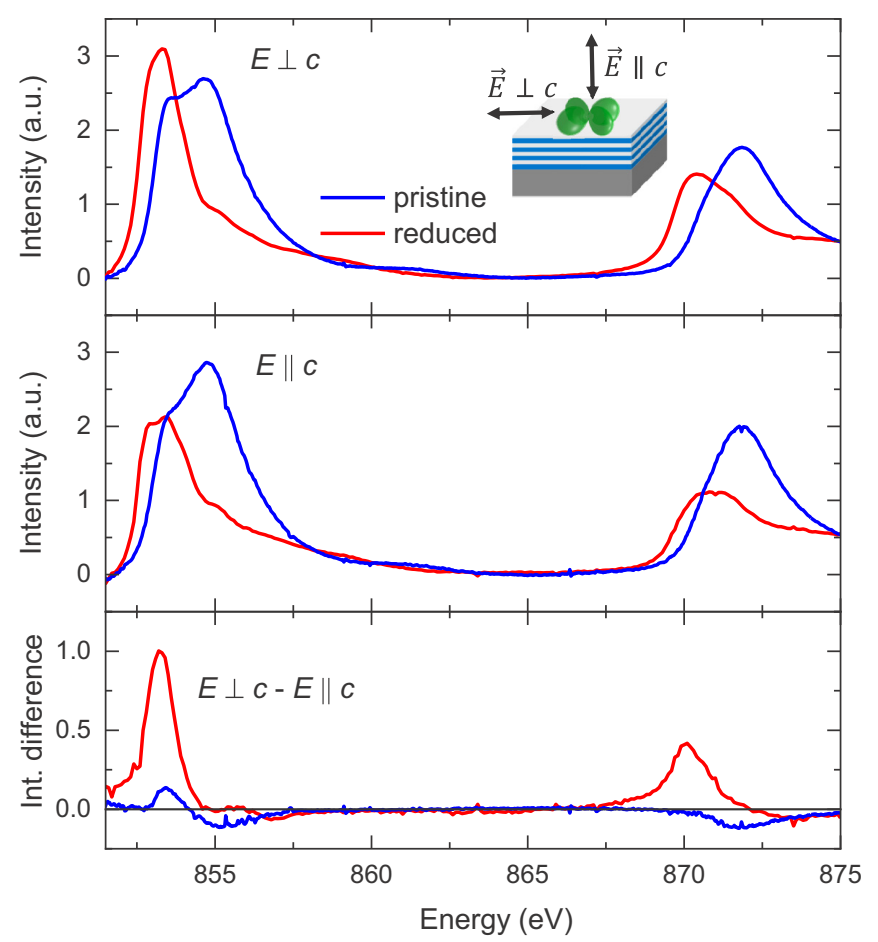

FIG. 5. Polarization-dependent XAS, measured in totalfluorescence yield for pristine and reduced samples across the Ni $L_{3,2}$ absorption edges, where the La $M_{4}$ lines were previously subtracted. The top and middle panels show the spectra taken with x-ray polarization $E \perp c$ and $E \| c$, respectively. A sketch of the geometry is shown in the inset of the top panel. The bottom panel shows the linear dichroism defined by the difference of intensities $I_{E \perp c}-I_{E \| c}$.

high- $T_{\mathrm{c}}$ cuprates, albeit reduced in size [34]. This quantitative difference is possibly related to the unevenly distributed charge across the $\mathrm{LaNiO}_{2+\delta}$ layer stack. As we will explain in the following section, the $a b$ initio calculations indicate that the self-doped holes at the interface are trapped and contribute with a strongly reduced linear dichroism to the layer-averaged spectra.

\section{THEORY: MODEL AND METHODS}

Transition-metal oxides have partially filled shells of rather localized orbitals and are therefore dominated by strong correlation effects. To include such effects (e.g., the opening of a Mott insulating gap), one possible strategy is the use of appropriate DFT or hybrid functionals (for recent applications, see, e.g., Refs. $[35,36])$. Here, however, we performed ab initio density functional theory (DFT) plus dynamical mean-field theory (DMFT) calculations-based on low-energy effective Hamiltonians- to gain further insight into the layerand orbital-resolved correlated electronic structure. We relaxed the ionic positions of the reduced unit-cell shown on the right-hand side in Fig. 1 with the Vienna $\mathrm{Ab}$ initio Simulation Package (VASP) code [37] using the generalized gradient approximation (GGA) functional [38]. Subsequent DFT self-consistency was performed using the full-potential local-orbital (FPLO) code [39,40]. For the Brillouin zone (BZ) integration, we used the tetrahedron method with a 
(a)

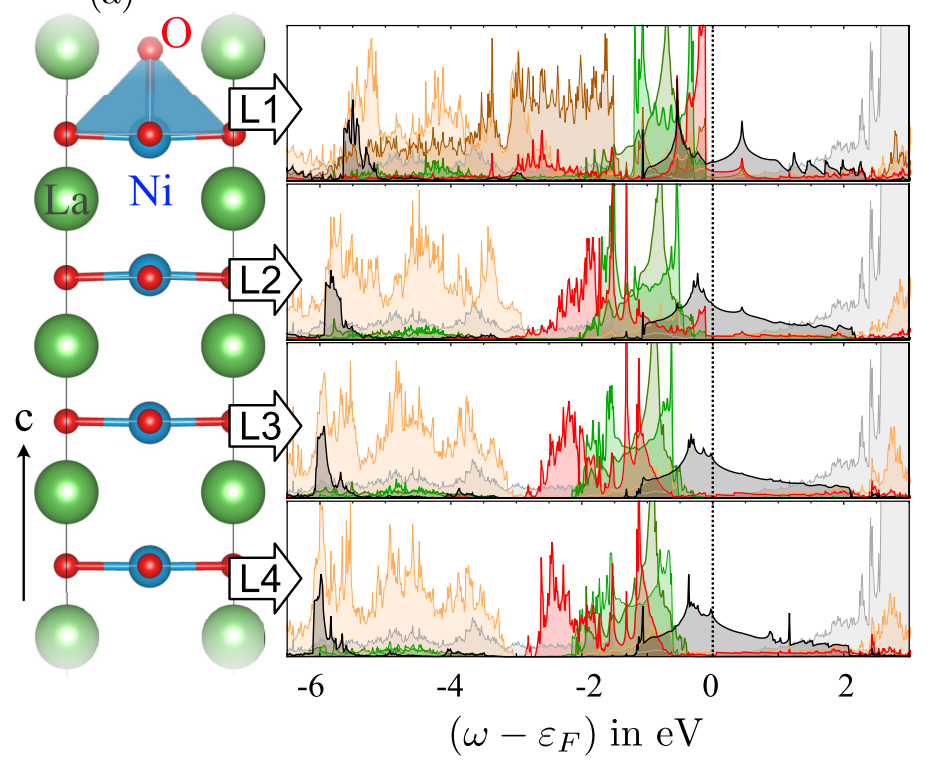

Ni 3d

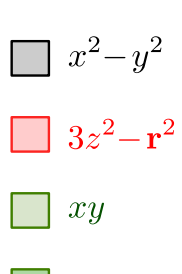

$x z / y z$

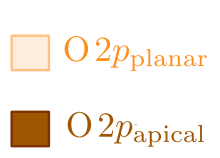

$\operatorname{La} 5 d$ (b)

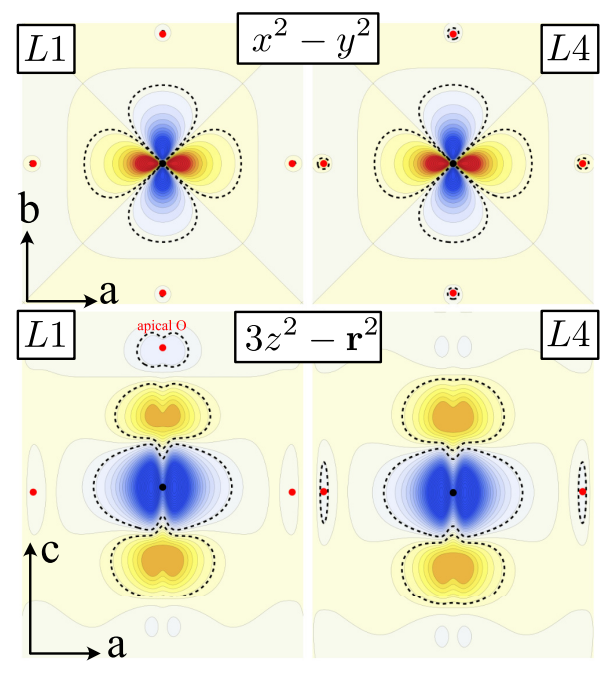

FIG. 6. (a) Partial density of states of the inequivalent $\mathrm{LaNiO}_{2+\delta}$ layers in the eight-layer stack with respect to electronic orbitals near the Fermi level (except for the La $5 d$ pDOS, which is averaged over all La sites) from DFT calculations. (b) Contour plot of Wannier functions with planar $x^{2}-y^{2}$ symmetry in the $a b$-plane (upper panels), and axial $3 z^{2}-\mathbf{r}^{2}$ symmetry in the $a c$-plane (lower panels) for interface- ( $L 1$ ) and innermost layer $(L 4)$. Black (red) dots indicate the position of $\mathrm{Ni}(\mathrm{O})$ ions. The dashed line marks the contour value of 0.05 .

$12 \times 12 \times 1 \mathbf{k}$-mesh. For the DMFT calculations, we downfolded the converged Kohn-Sham eigenstates to a basis of 195 orbitals (per spin) including (i) the $4 s$ - and $3 d$-shells of all $\mathrm{Ni}$ ions, (ii) the $2 p$-shell of all $\mathrm{O}$ ions, and (iii) the $5 d$-shell of all $\mathrm{La}$ ions. We note that especially the explicit treatment of $\mathrm{Ni} 4 s$ as well as La $5 d$ degrees of freedom significantly improved the localization of the effective $3 d$ Wannier functions. In Fig. 6(b) we show examples of localized $d_{x^{2}-y^{2}}$ and $d_{3 z^{2}-\mathbf{r}^{2}}$ Wannier orbitals at the interface $L 1$ and the inner layer $L 4$.

The dynamical mean-field calculations were performed by converging four (one for each nonequivalent Ni site) auxiliary impurity problems in full charge self-consistency. Each one of the impurity problems contains the full $3 d$ shell of the respective $\mathrm{Ni}$ ion and was solved for a fully $\mathrm{SU}(2)$ symmetric Kanamori operator with Hubbard $U=8.0 \mathrm{eV}$, Hund's coupling $J_{H}=0.8 \mathrm{eV}$, and the assumption of fixed interorbital interaction $U^{\prime}=U-2 J_{H}[41,42]$, which is a common approximation for the full local Coulomb operator (see Appendix B for the full Hamiltonian). These specific values for the interaction parameters were chosen in order to reproduce quasiparticle renormalization factors comparable to previous LDA + DMFT calculations [43], where $U$ and $J$ were computed by the constrained random-phase approximation. We remark that to achieve this, our $U$ and $J$ parameters needed to be enlarged compared to Ref. [43] due to the explicit inclusion of $\mathrm{Ni} 4 s$ and oxygen $2 p$ orbitals in the DMFT calculation. For our calculations, we used the W2DYNAMICS code [44] and its associated continuous-time quantum Monte Carlo solver in the hybridization expansion version. To account for the appearance of the Hartree term in both DFT and DMFT, we employ the "fully localized limit" double-counting correction [45]. All calculations were performed at a temperature of $T=1 / 50\left(\mathrm{eV}^{-1}\right) \approx 232 \mathrm{~K}$. Following common procedure, the total charge of the lattice model remains fixed within the DMFT self-consistent calculation, which allows for a well-defined simulation of doping in the correlated system. All calculations were performed in the paramagnetic phase, i.e., magnetic order is suppressed to overcome the ordering tendency inherent to a spatial mean-field method like DMFT. The analytical continuation to calculate the DMFT spectral function shown in Fig. 7 was performed by using the maximum entropy method (MaxEnt) as implemented in Ref. [46]. Further calculation details can be found in Ref. [47].

\section{NONINTERACTING REFERENCE MODEL}

We start our discussion on the level of the noninteracting reference model derived from density functional theory. In Fig. 6(a) we show the layer- and orbitally resolved partial density of states (pDOS) around the Fermi level $\left(\varepsilon_{F}\right)$. We find for all layers the Ni $3 d$ states to be partially filled and in-between the $\mathrm{O} 2 p$ and the La $5 d$ states. Another common observation for all layers is that the $\mathrm{Ni} d_{x^{2}-y^{2}}$ weight is the most prominent at and above $\varepsilon_{F}$. Even though we find some of the $d_{x^{2}-y^{2}}$ weight at around $-6 \mathrm{eV}$ in a covalent bond with oxygen, its overall hybridization with the $\mathrm{O} 2 p$ states is rather weak (e.g., in comparison to charge-transfer systems like high- $T_{c}$ cuprates). This is in accordance with the experimental $\mathrm{O} K$ edge spectra discussed in Sec. III.

A closer look at the pDOS in Fig. 6(a) below $\varepsilon_{F}$ reveals significant differences between the electronic structure of interface $L 1$ and the inner layers $L 2-L 4$. This is due to ligand-field effects involving the apical oxygen at the interface and the apical oxygen vacancies in the inner layers. Due to its extension along the $c$-axis, it is not surprising that the $d_{3 z^{2}-\mathbf{r}^{2}}$ orbitals are affected the most. The strong $\sigma$-hybridization with the apical oxygen states in the interface layer $L 1$ leads to a 




FIG. 7. Layer- $(L 1-L 4)$ and orbitally resolved k-integrated spectral function $\mathrm{A}(\omega)$ from DMFT for interaction values $U=8 \mathrm{eV}$ and $J=$ $0.8 \mathrm{eV}$ at $T=1 / 50\left(\mathrm{eV}^{-1}\right) \approx 232 \mathrm{~K}$. The columns correspond to different total electron fillings, where negative (positive) $\Delta N$ corresponds to hole (electron) doping. The insets show the orbital-dependent real part of the DMFT self-energy $\Sigma^{\prime}\left(i \omega_{n}\right)$ on the Matsubara frequency axis $\left[\omega_{n}=(2 n+1) \pi k_{B} T, n \in \mathbb{Z}\right]$. The energy range and the color code for the plots are identical to the ones used in Fig. 6.

large peak between -1.0 and $-0.5 \mathrm{eV}$ corresponding to the antibonding combination of $d_{3 z^{2}-\mathbf{r}^{2}}$ and apical oxygen $2 p_{z}$. As a secondary effect, driven by nonlocal hybridization of the axial state with the planar $d_{x^{2}-y^{2}}$ orbitals, we also find an additional peak in the $d_{x^{2}-y^{2}}$ pDOS in the interface layer $L 1$ at about $-1 \mathrm{eV}$, which is absent in the inner layers. Interfacial $L 1: d_{x z} / d_{y z}$ orbitals are also affected by the apical oxygen (albeit on a smaller energy scale) due to $\pi$-hybridization with apical $p_{x}$ - and $p_{y}$-orbitals, which brings them closer to $\varepsilon_{F}$ for the interface layer.

The apical oxygen vacancies in the inner layers lead to a different ligand-field mechanism for the $d_{3 z^{2}-\mathbf{r}^{2}}$ states. We observe that the oxygen vacancy is not simply an "absence of oxygen" but provokes the extension of especially the La $5 d$ orbitals into the vacancy site. Their probability density at the vacancy site is large enough that a single-particle basis transformation to a "bondlike" state, which has been referred to as "zeronium" orbital [48], leads to an insightful perspective: when apical oxygen orbitals (which are full in the ionic limit) are replaced by zeronium orbitals (which are empty in the ionic limit), the $d_{3 z^{2}-\mathbf{r}^{2}}$ states around $\varepsilon_{F}$ no longer form antibonding states with apical oxygen $2 p_{z}$, but instead they form bonding zeronium- $d_{3 z^{2}-\mathbf{r}^{2}}$ states, which is reflected in a dramatic lowering of the corresponding $d_{3 z^{2}-\mathbf{r}^{2}}$ pDOS in the inner layers and a small but non-negligible increase in the weight of $d_{3 z^{2}-\mathbf{r}^{2}}$ to energies well above $\varepsilon_{F}$. Interestingly, the electronic structure of the interface $\mathrm{Ni}$ site (in $L 1$ ) shows features originating from both ligand-field effects, the one with apical oxygen and the one with zeronium ligand.

The layer-dependent ligand field effects are also reflected in the orbital occupations projected into the $\mathrm{Ni} 3 d$ subspace listed in Table I of Appendix D. The small difference in total $3 d$ occupations of central and interface layers of only $n_{\text {tot }}(L 4)-n_{\text {tot }}(L 1)=0.06$ might seem surprising in light of the strong layer dependence of the pDOS. The reason for this small difference is a "damping" of the density differences arising from the aforementioned ligand field effects: Expanding the electronic configurations around the ionic limit with a layer-dependent ansatz looks like this:

$$
\left|\Psi_{L 1}\right\rangle=\alpha_{1}\left(\mathrm{Ni} 3 d^{8}\right)+\beta_{1}\left(\mathrm{Ni} 3 d^{9} \underline{L}\right)+\gamma_{1}\left(\mathrm{Ni} 3 d^{7} z\right)+\cdots
$$

for the interface and

$$
\begin{aligned}
\left|\Psi_{L 2-L 4}\right\rangle= & \alpha_{2-4}\left(\mathrm{Ni} 3 d^{9}\right)+\beta_{2-4}\left(\mathrm{Ni} 3 d^{10} \underline{L}\right) \\
& +\gamma_{2-4}\left(\mathrm{Ni} 3 d^{8} z\right)+\cdots
\end{aligned}
$$

for the inner layers. Here $\underline{L}$ denotes an (oxygen) ligand-hole and $z$ denotes a (zeronium) ligand electron. The hybridization with apical oxygen at the interface layer increases the Ni-projected charge due to an enhancement of $\beta$ (i.e., the formation of oxygen holes), while hybridization with zeronium in the inner layers enhances $\gamma$ and thus depletes it. The density analysis shows the limited value of projected density operators and underlines the need to use a configuration interaction language rather than "occupations."

In summary, we find strong and qualitative differences of the electronic structure for orbitals with extension along the $c$-axis. The most relevant mechanism is the (covalent) ligand-field effects for the $d_{3 z^{2}-\mathbf{r}^{2}}$ orbital whose spectral weight is pushed towards the Fermi level in $L 1$ due to the apical oxygen and pushed away from the Fermi level in $L 2-L 4$ due to its overlap with zeronium states. We will now see that these layer-dependent orbital polarization effects on the single-particle level are even further enhanced when electronic interactions are considered beyond the static meanfield level. 


\section{DYNAMICAL MEAN-FIELD THEORY}

We start the discussion of our DMFT calculations [for $U=8 \mathrm{eV}$ and $J=0.8 \mathrm{eV}$ at $\left.T=1 / 50\left(\mathrm{eV}^{-1}\right) \approx 232 \mathrm{~K}\right]$ with the layer- and orbital-resolved single-particle spectral functions, which are shown in Fig. 7 for different values of total filling ( $\Delta N=0$ corresponds to the reduced heterostructure as displayed in Fig. 1 without any extra charge).

\section{A. Nominal doping}

Comparing the DMFT spectra of the system at nominal doping $(\Delta N=0)$ to the partial DOS from DFT in Fig. 6 reveals two major effects originating from local correlations: (i) a renormalization of the quasiparticle $(\mathrm{QP})$ masses, and (ii) an enhancement of the layer-dependent orbital polarizations. Effect (i) is the expected sharpening of the spectra at the Fermi level. The corresponding (orbital-dependent) mass (m) renormalization $Z_{\alpha} \equiv m_{\alpha}^{\mathrm{QP}} / m_{\alpha}^{\text {free }}$ depends on layer and orbital (index $\alpha$ ) and can be estimated from the self-energy (for details, see Appendix C). At nominal doping, the inner $d_{x^{2}-y^{2}}$ QPs have (4-5) $\times m^{\text {free }}$, while at the interface we find $2.5 \times m^{\text {free }}$. A closer look at the interface reveals also a second

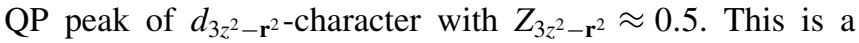
consequence of (ii), i.e., the enhanced layer-dependent orbital polarization of the Ni $3 d$ shell. In the interface layer $L 1$ the spectral weight of the $d_{3 z^{2}-\mathbf{r}^{2}}$ orbital is pushed across the Fermi level and is the dominant contribution to the sharp QP peak around $\varepsilon_{F}$. In the inner layers, in contrast (especially $L 3$ and $L 4$ ), the spectral weight of the $d_{3 z^{2}-\mathbf{r}^{2}}$ orbital remains well below $\varepsilon_{F}$, and the QP peak has almost pure $d_{x^{2}-y^{2}}$ character.

The results for the spectral weight transfer from the MaxEnt method are supported by data obtained directly on the Matsubara axis. The stochastic nature of the MaxEnt method always incurs an uncertainty for the analytical continuation, which is not present for quantities on the Matsubara axis, which are produced directly by the quantum Monte Carlo solver. In the insets of Fig. 7 we show the real part of the DMFT self-energy $\Sigma^{\prime}\left(i \omega_{n}\right)$ (with respect to the chemical potential $\mu=0$ ) for the correlated $d$-orbitals with error bars plotted on the Matsubara frequency axis (color code as for the spectral functions). The extrapolation of $\Sigma^{\prime}\left(i \omega_{n} \rightarrow 0\right)$ provides an estimate of the orbital-dependent spectral weight shifts at small energies. This self-energy driven spectral polarization changes sign from the interface (where the extrapolation of self-energy for the $d_{3 z^{2}-\mathbf{r}^{2}}$ orbital is $\approx 0.5 \mathrm{eV}$ larger than for the other orbitals) to the inner layers, where the $d_{x^{2}-y^{2}}$ weight is shifted up in energy instead. This means that local correlation effects enhance the tendencies of the single-particle DFT calculations. The origin of this enhancement is the Hund's coupling and has been studied in detail for generic $d p$-models in DMFT [49]. If orbitals are close in energy, additional Hund's energy can be gained by equalizing orbital occupations and maximizing total spin, which is what we observe at the interface [50]. For the inner layers, however, high-spin configurations are too high in energy to begin with, and Hund's coupling is less important. For completeness, the projected densities at the Ni site from DMFT calculations are provided in Table II of Appendix D.

\section{B. Hole and electron doping}

To improve our understanding, we consider the effect of extra charge in the heterostructure. To this end, we consider the addition of two and four holes or electrons per unit cell, i.e., a change in the total number of electrons of $\Delta N \in\{-4,-2,2,4\}$ in comparison to the nominal doping $\Delta N=0$. The corresponding spectra are shown in Fig. 7 . For the inner layers $L 2-L 4$ we find singe-band behavior: doping the $d_{x^{2}-y^{2}}$ band away from half-filling decreases the mass-renormalization of the QPs (see Z-factors in Fig. 10 of Appendix C). The same is true also for the interface layer $L 1$ in the electron-doped case.

The spectra of the interface layer on the hole-doped side are, however in stark contrast to this correlated single-band picture: For $\Delta N=-2$ we observe in the interface layer an increased transfer of spectral weight of the $d_{3 z^{2}-\mathbf{r}^{2}}$ orbital and an even larger splitting in the real part of the self-energy to the $d_{x^{2}-y^{2}}$ orbital compared to the nominal doping $\Delta N=0$ case. Due to this shift, a large amount of the extra holes is trapped at the interface. This is also true for even larger hole-doping $\Delta N=-4$, where instead of an even distribution across the layers, the holes migrate to interface $d_{x z / y z}$ orbitals.

To understand the mechanism leading to the trapping of holes in the interface layer, we provide histograms of the layer- and orbital-resolved projected densities at each Ni site, relative to the value at nominal doping in Fig. 8(a) and of the configurations of the Ni local spin moments in Fig. 8(b). On the electron-doped side, the density histograms in Fig. 8(a) confirm that the additional electrons distribute evenly across layers. On the hole-doped side, in contrast, the extra carriers are trapped at the interface layers initially in the $d_{3 z^{2}-\mathbf{r}^{2}}$ orbital and then in the $d_{x z / y z}$ orbital, once the $d_{3 z^{2}-\mathbf{r}^{2}}$ orbital has become half-filled. The reason for this trapping can be understood by considering the spin configurations of the local Ni moment in Fig. 8(b). For $\Delta N=-2$, the center of the configuration distribution shifts from $S=1 / 2$ to 1 , which is representative of the formation of the high-spin state in the $d_{3 z^{2}-\mathbf{r}^{2}}$ orbital to gain Hund's coupling energy. At $\Delta N=-4$ the maximum at $S=1$ becomes more pronounced, and we observe a finite weight of $S=3 / 2$ configurations (consistent with added holes of $d_{x z / y z}$ character). The true many-body nature of this local moment formation is also reflected in the mass of interface $L 1$ QPs: instead of a decreased mass renormalization (like we observe for the inner layers), the Hund's correlation dominated $L 1$ QPs show $Z$-factors as low as $Z_{3 z^{2}-\mathbf{r}^{2}} \approx 0.06$ (i.e., a QP mass enhancement by a factor $\approx 16.6$ ) as estimated from Fig. 10. This observation indicates the creation of a strongly correlated Hund's metal [51] in the $L 1$ interface, which traps most of the doped holes. Finally, we note that the distribution of the extra charge into $\mathrm{O} 2 p$ and La $5 d$ states is negligible.

In summary, our theoretical analysis indicates that the supposedly homogeneously self-doped heterostructure has a strongly layer-dependent electronic structure. Ligand-field effects due to apical oxygen and apical vacancies on the single-particle level are enhanced by local correlation effects. Careful analysis of spectral functions, projected densities, many-body configuration histograms, and the dynamic selfenergies indicate the formation of a strongly correlated 

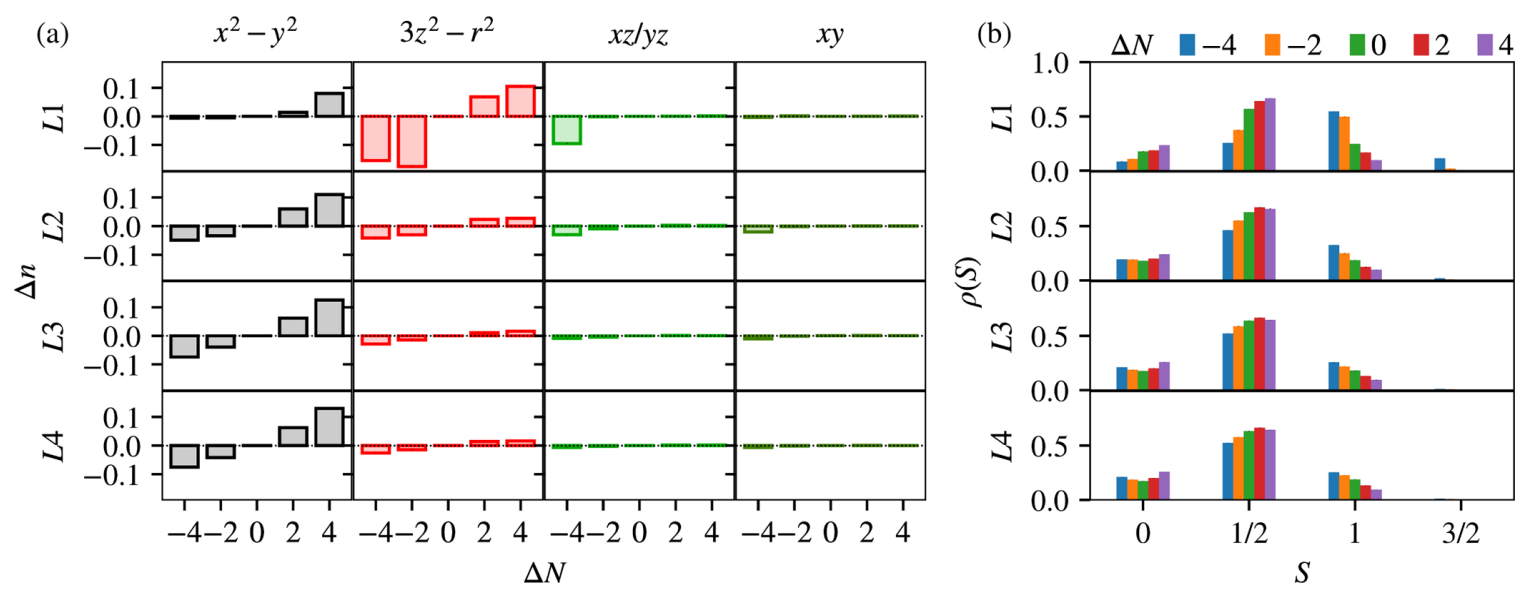

FIG. 8. (a) Layer- and orbital-resolved distribution of the density $\Delta n$ at the inequivalent Ni sites. (b) Layer-resolved spin density $\rho(S)$ as determined by projecting the many-particle density matrix into the basis of the $S^{2}$ operator.

Hund's metal at the interface layer. In the concomitant formation of local high-spin configurations, doped holes are trapped at the interface, rather than being distributed evenly across the heterostructure. In contrast, doped electrons are distributed evenly across the layers, filling up $d_{x^{2}-y^{2}}$ states.

\section{SUMMARY AND CONCLUSIONS}

In summary, we have shown that reversible, layer-selective removal of oxygen ions yields $\mathrm{LaNiO}_{2+\delta}-\mathrm{LaGaO}_{3}$ superlattices with well-ordered infinite-layer nickelate stacks, and we used x-ray diffraction and transmission electron microscopy to characterize the structure. Based on our combined results from electrical transport, x-ray spectroscopy, and ab initio calculations, we draw the following conclusions: (i) The oxygen reduction of the superlattice is accompanied by a change in the electronic structure, which is characterized by a transition from a negative charge-transfer to a Mott-Hubbard type system. The shift of the $\mathrm{O} p$ derived bands to energies well below the occupied Ni $d$ states in DFT + DMFT is supported by our observations from $\mathrm{O} K$ edge XAS, where the $d^{8} \underline{L} \rightarrow d^{8}$ prepeak of the pristine samples is completely suppressed, and the only remaining $d^{9} \underline{L} \rightarrow d^{10}$ transitions in reduced samples are weak and hidden under the continuum part of the spectrum. Experiments on undoped infinite-layer $\mathrm{LaNiO}_{2}$ thin films came to a similar conclusion [23]. (ii) The sharp peaks at $\varepsilon_{F}$ in the DMFT spectra indicate the presence of heavy quasiparticles. The small amount of coherent spectral weight and sizable mass-enhancement factors indicates that the system is very close to an insulating state, which is consistent with the experimental findings of a semiconducting electronic transport behavior. Either additional electronic correlations that were not taken into account in our DFT + DMFT calculations can lead to a Mott-isolating state, or localization is caused by residual disorder in the experimentally realized superlattices. A combination of both effects is equally possible. (iii) The layer-averaged, overall dominant $\mathrm{Ni} d_{x^{2}-y^{2}}$ orbital character of the electron-addition states calculated in DMFT qualitatively agrees with the spectral shape and sign of the observed linear dichroism measured by $\mathrm{Ni} L$ edge XAS. For the inner layers $L 2-L 3$, the DMFT results are reminiscent of the single-band Hubbard model that possibly hosts superconductivity upon doping. (iv) Despite having a very similar self-doping level to the superconducting $\mathrm{Nd}_{0.8} \mathrm{Sr}_{0.2} \mathrm{NiO}_{2}$ films reported in Ref. [1], the superlattices do not exhibit superconductivity down to 2 K (Fig. 2). The electronic-structure calculations yield an explanation for this striking difference in terms of Hund's coupling stabilized trapping of the self-doped holes at the superlattice interfaces. Based on this understanding, the DMFT results provide a promising outlook for this superlattice structure upon electron-doping, where a uniform distribution of the doped electrons across the nickelate layer stack should be possible. More generally, our combined experimental and theoretical results provide an interesting perspective for the interfacial doping approach of transition-metal oxide superlattices.

\section{ACKNOWLEDGMENTS}

We acknowledge financial support by the Center for Integrated Quantum Science and Technology $\left(\mathrm{IQ}^{\mathrm{ST}}\right)$ and the Deutsche Forschungsgemeinschaft (DFG, German Research Foundation): Projektnummer 107745057-TRR 80 and Projektnummer 323667265. We thank HZB for the allocation of synchrotron radiation beamtime. We thank G. A. Sawatzky, K. Foyevtsova, R. Merkle, and M. Klett for inspiring and very insightful discussions. We thank Manuel Mundszinger for FIB sample preparation.

\section{APPENDIX A: HRTEM MEASUREMENTS}

Cross-sectional HRTEM specimens of pristine and reduced samples from the same growth [Figs. 9(a) and 9(b)] were prepared by the focused ion beam (FIB) technique using a NVision 040 Ar with a special X2-holder [52] for high-quality lamella preparation. We chose pseudocubic $(1-10)_{\mathrm{pc}}$ plane cuts to determine accurately the atomic column distances along the superlattice growth direction. Imaging-side spherical aberration-corrected (AC)-FEI Titan 80-300 kV was used in HRTEM imaging mode operated at $300 \mathrm{kV}$. Negativespherical-aberration $\left(\mathrm{C}_{s}\right)$ imaging conditions $\left(\mathrm{C}_{s}=-15 \mu \mathrm{m}\right)$ were applied to image the atomic columns by white atom contrast [53]. For the quantitative analysis, the AC-HRTEM images were filtered by Wiener filter to enhance the signal- 


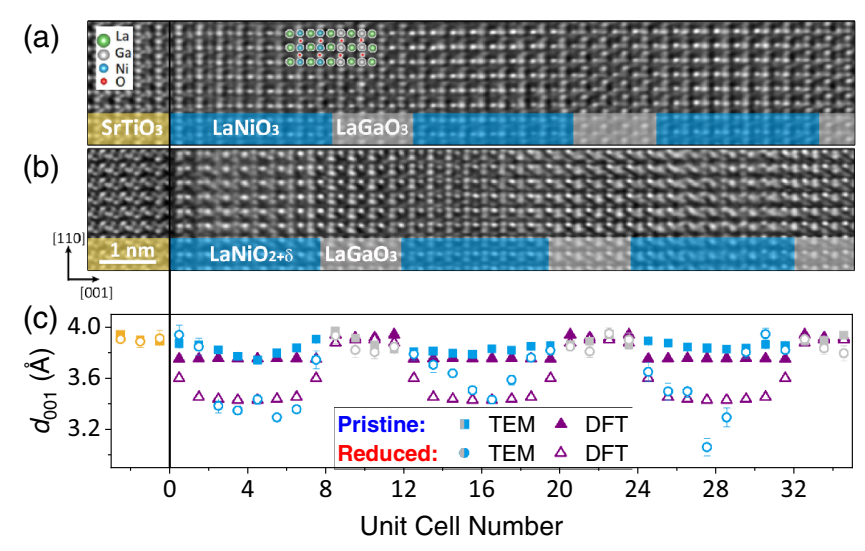

FIG. 9. Negative- $C_{s}$ HRTEM images of the $m=8, n=4$ (a) pristine and (b) reduced superlattices measured in $[1-10]_{\mathrm{pc}}$ projection. The interfaces between $\mathrm{SrTiO}_{3}$ substrate (yellow) and $\mathrm{LaNiO}_{3(2+\delta)}$ (blue) $-\mathrm{LaGaO}_{3}$ (gray) layer stacks are clearly shifted in the reduced sample due to the $d_{001}$ contraction upon oxygen removal. Schematic atomic model is overlaid on the HRTEM image in panel (a). (c) Values of the $d_{001}$-spacing in pristine and reduced superlattices measured from the HRTEM images (a,b), where each data point represents the average value of 15 unit cells along the $[110]_{\mathrm{pc}}$ axis. For comparison we show the corresponding values from the DFT structure relaxation.

to-noise ratio. CalAtom [54] together with a self-written MATLAB code was used to extract the lattice parameter values $d_{001}$ [Fig. 9(c)] within the individual $\mathrm{LaNiO}_{3(2+\delta)}$ and $\mathrm{LaGaO}_{3}$ layer stacks along the superlattice growth direction. To distinguish $\mathrm{LaNiO}_{3(2+\delta)}$ from the $\mathrm{LaGaO}_{3}$ layer stacks, we took advantage of the different pattern that results from the Pbnm distortion of $\mathrm{LaGaO}_{3}$ in the selected projection [55].
Together with the information on the stacking sequence from the growth (starting with eight unit cells of $\mathrm{LaNiO}_{3(2+\delta)}$ from the substrate, followed by four unit cells of $\mathrm{LaGaO}_{3}$, and so on) we identified the interfaces. Within the experimental accuracies of the methods, we found good agreement between TEM and XRD data and the corresponding DFT $d_{001}$ values. The latter were derived by relaxing the ionic positions within the superlattice unit cell, whereby the in-plane lattice parameter and the bilayer thickness were fixed to the experimental XRD values of pristine and reduced superlattices, respectively.

\section{APPENDIX B: HUBBARD-KANAMORI HAMILTONIAN}

The local interaction Hamiltonian in Hubbard-Kanamori form reads

$$
\begin{aligned}
H_{\mathrm{int}}= & U \sum_{m} \hat{n}_{m, \uparrow} \hat{n}_{m, \downarrow} \\
& +U^{\prime} \sum_{m \neq m^{\prime}} \hat{n}_{m^{\prime}, \uparrow} \hat{n}_{m^{\prime}, \downarrow} \\
& +\left(U^{\prime}-J_{H}\right) \sum_{m<m^{\prime}, \sigma} \hat{n}_{m, \sigma} \hat{n}_{m^{\prime}, \sigma} \\
& +J_{H} \sum_{m \neq m^{\prime}} \hat{d}_{m, \uparrow}^{\dagger} \hat{d}_{m^{\prime}, \downarrow}^{\dagger} \hat{d}_{m, \downarrow} d_{m^{\prime}, \uparrow} \\
& +J_{H} \sum_{m \neq m^{\prime}} \hat{d}_{m, \uparrow}^{\dagger} \hat{d}_{m, \downarrow}^{\dagger} \hat{d}_{m^{\prime}, \downarrow} d_{m^{\prime}, \uparrow},
\end{aligned}
$$

where (B1a) is the intraorbital Coulomb interaction, (B1b) and (B1c) are the interorbital Coulomb interaction, (B1d) is

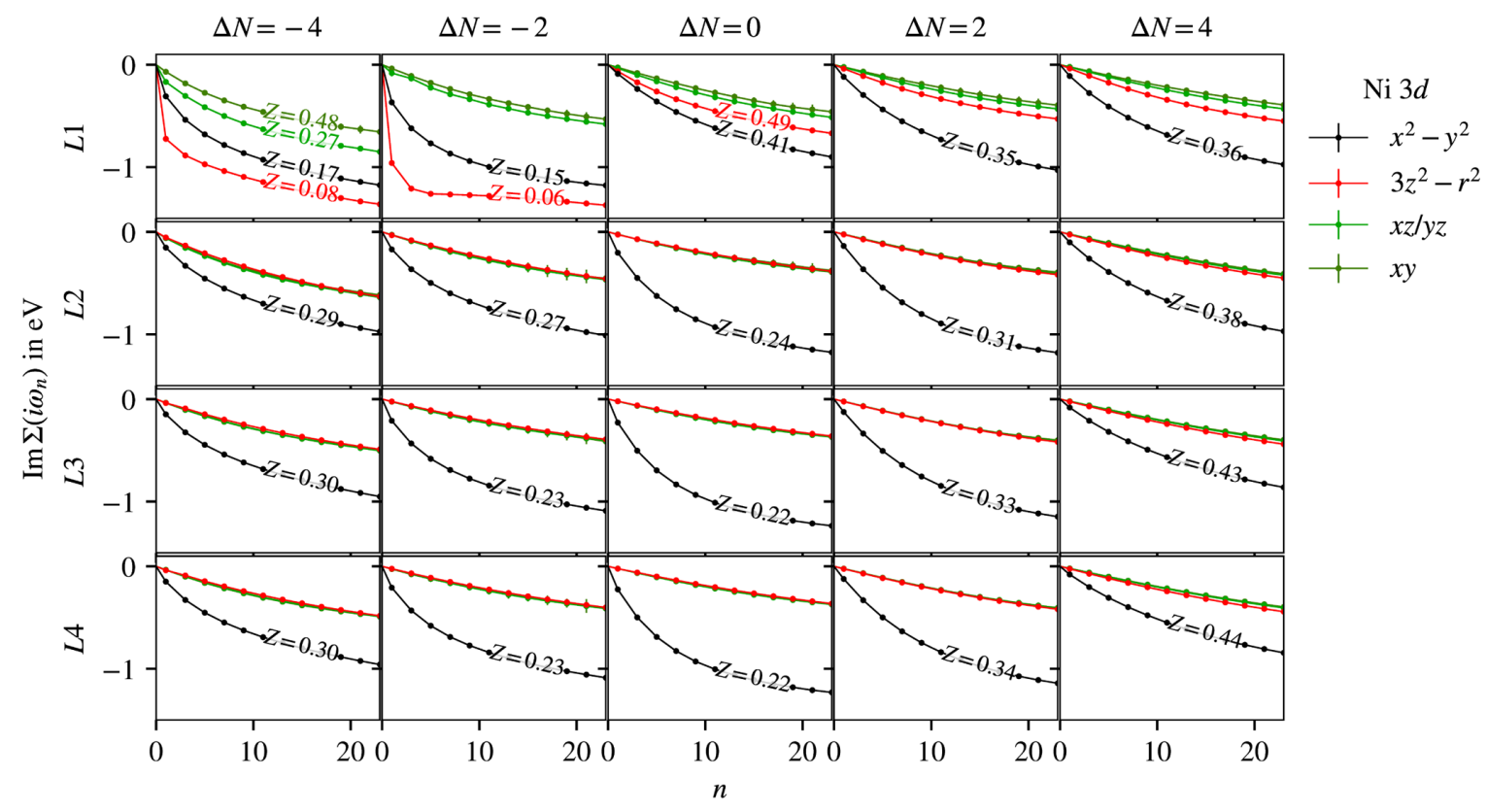

FIG. 10. Layer- and orbital-resolved imaginary part of the self-energy. The lines are annotated with the corresponding quasiparticle renormalization factor where there is a quasiparticle peak present in Fig. 7. 
TABLE I. Layer-resolved projected DFT densities of the Ni $3 d$ orbitals (per spin).

\begin{tabular}{lccccc}
\hline \hline & $x^{2}-y^{2}$ & $3 z^{2}-\mathbf{r}^{2}$ & $x z / y z$ & $x y$ & $n_{\text {tot }}$ \\
\hline$L 1$ & 0.57 & 0.81 & 0.97 & 0.98 & 4.31 \\
$L 2$ & 0.59 & 0.83 & 0.97 & 0.98 & 4.35 \\
L3 & 0.61 & 0.84 & 0.96 & 0.98 & 4.37 \\
L4 & 0.61 & 0.84 & 0.96 & 0.98 & 4.37 \\
\hline \hline
\end{tabular}

the Hund's rule spin-flip interaction, and (B1e) is the Hund's rule pair-hopping interaction.

\section{APPENDIX C: DMFT SELF ENERGIES}

In Fig. 10 we show the imaginary part of the layer- and orbital-resolved self-energy from which we can extract the QP renormalization factor for the spectra in Fig. 7. The mass renormalization factor is defined as

$$
Z_{\alpha} \equiv m_{\alpha}^{\mathrm{QP}} / m_{\alpha}^{\text {free }} \approx \frac{1}{1-\operatorname{Im} \Sigma_{\alpha}^{\mathrm{DMFT}}\left(i \omega_{0}\right) / \omega_{0}}
$$

TABLE II. DMFT densities (per spin).

\begin{tabular}{lccccc}
\hline \hline & $x^{2}-y^{2}$ & $3 z^{2}-\mathbf{r}^{2}$ & $x z / y z$ & $x y$ & $n_{\text {tot }}$ \\
\hline$L 1$ & 0.60 & 0.78 & 0.98 & 0.99 & 4.34 \\
$L 2$ & 0.57 & 0.86 & 0.98 & 0.99 & 4.39 \\
$L 3$ & 0.58 & 0.87 & 0.97 & 0.99 & 4.40 \\
$L 4$ & 0.58 & 0.87 & 0.97 & 0.98 & 4.40 \\
\hline \hline
\end{tabular}

where $\omega_{0}=\pi k_{B} T$ is the first fermionic Matsubara frequency defined by the Boltzmann constant $k_{B}$ and temperature $T$, and $\Sigma_{\alpha}^{\text {DMFT }}$ is the local DMFT self-energy for orbital $\alpha$. We only provide renormalization factors for those orbitals that contribute to the coherent QP peak at the Fermi energy.

\section{APPENDIX D: DFT AND DMFT DENSITIES}

Table I shows the layer-dependent ligand field effects, which are reflected in the orbital occupations projected into the Ni $3 d$ subspace. The projected densities at the Ni site from DMFT calculations are provided in Table II.
[1] D. Li, K. Lee, B. Y. Wang, M. Osada, S. Crossley, H. R. Lee, Y. Cui, Y. Hikita, and H. Y. Hwang, Nature (London) 572, 624 (2019).

[2] S. Zeng, C. S. Tang, X. Yin, C. Li, M. Li, Z. Huang, J. Hu, W. Liu, G. J. Omar, H. Jani, Z. S. Lim, K. Han, D. Wan, P. Yang, S. J. Pennycook, A. T. S. Wee, and A. Ariando, Phys. Rev. Lett. 125, 147003 (2020).

[3] D. Li, B. Y. Wang, K. Lee, S. P. Harvey, M. Osada, B. H. Goodge, L. F. Kourkoutis, and H. Y. Hwang, Phys. Rev. Lett. 125, 027001 (2020).

[4] M. Osada, B. Y. Wang, K. Lee, D. Li, and H. Y. Hwang, Phys. Rev. Mater. 4, 121801(R) (2020).

[5] K. Lee, B. H. Goodge, D. Li, M. Osada, B. Y. Wang, Y. Cui, L. F. Kourkoutis, and H. Y. Hwang, APL Mater. 8, 041107 (2020).

[6] M. Crespin, P. Levitz, and L. Gatineau, J. Chem. Soc., Faraday Trans. 2 79, 1181 (1983).

[7] P. Levitz, M. Crespin, and L. Gatineau, J. Chem. Soc., Faraday Trans. 2 79, 1195 (1983).

[8] M. A. Hayward, M. A. Green, M. J. Rosseinsky, and J. Sloan, J. Am. Chem. Soc. 121, 8843 (1999).

[9] M. Crespin, O. Isnard, F. Dubois, J. Choisnet, and P. Odier, J. Solid State Chem. 178, 1326 (2005).

[10] Y. Shimakawa, S. Inoue, M. Kawai, N. Ichikawa, M. Mizumaki, and N. Kawamura, MRS Proc. 1148, 708 (2008).

[11] M. Kawai, S. Inoue, M. Mizumaki, N. Kawamura, N. Ichikawa, and Y. Shimakawa, Appl. Phys. Lett. 94, 082102 (2009).

[12] D. Kaneko, K. Yamagishi, A. Tsukada, T. Manabe, and M. Naito, Physica C 469, 936 (2009), Proceedings of the 21st International Symposium on Superconductivity (ISS 2008).

[13] T. Moriga, O. Usaka, I. Nakabayashi, T. Kinouchi, S. Kikkawa, and F. Kanamaru, Solid State Ion. 79, 252 (1995), Proceedings of the 20th Commemorative Symposium on Solid State Ionics in Japan.

[14] F. Lechermann, Phys. Rev. B 101, 081110(R) (2020).
[15] F. Bernardini and A. Cano, J. Phys. Mater. 3, 03LT01 (2020).

[16] A. S. Botana and M. R. Norman, Phys. Rev. X 10, 011024 (2020).

[17] Y. Zhang, L.-F. Lin, W. Hu, A. Moreo, S. Dong, and E. Dagotto, Phys. Rev. B 102, 195117 (2020).

[18] V. M. Katukuri, N. A. Bogdanov, O. Weser, J. van den Brink, and A. Alavi, Phys. Rev. B 102, 241112(R) (2020).

[19] G. Sawatzky, Nature (London) 572, 592 (2019).

[20] J. Q. Lin, P. Villar Arribi, G. Fabbris, A. S. Botana, D. Meyers, H. Miao, Y. Shen, D. G. Mazzone, J. Feng, S. G. Chiuzbăian, A. Nag, A. C. Walters, M. García-Fernández, K.-J. Zhou, J. Pelliciari, I. Jarrige, J. W. Freeland, J. Zhang, J. F. Mitchell, V. Bisogni, X. Liu, M. R. Norman, and M. P. M. Dean, Phys. Rev. Lett. 126, 087001 (2021).

[21] H. Lu, M. Rossi, A. Nag, M. Osada, D. F. Li, K. Lee, B. Y. Wang, M. Garcia-Fernandez, S. Agrestini, Z. X. Shen, E. M. Been, B. Moritz, T. P. Devereaux, J. Zaanen, H. Y. Hwang, K.-J. Zhou, and W. S. Lee, Science 373, 213 (2021).

[22] K.-W. Lee and W. E. Pickett, Phys. Rev. B 70, 165109 (2004).

[23] M. Hepting, D. Li, C. J. Jia, H. Lu, E. Paris, Y. Tseng, X. Feng, M. Osada, E. Been, Y. Hikita, Y.-D. Chuang, Z. Hussain, K. J. Zhou, A. Nag, M. Garcia-Fernandez, M. Rossi, H. Y. Huang, D. J. Huang, Z. X. Shen, T. Schmitt, H. Y. Hwang, B. Moritz, J. Zaanen, T. P. Devereaux, and W. S. Lee, Nat. Mater. 19, 381 (2020).

[24] K. Matsumoto, M. Haruta, M. Kawai, A. Sakaiguchi, N. Ichikawa, H. Kurata, and Y. Shimakawa, Sci. Rep. 1, 27 (2011).

[25] B. Geisler and R. Pentcheva, Phys. Rev. B 102, 020502(R) (2020).

[26] J. Zhang, A. S. Botana, J. W. Freeland, D. Phelan, H. Zheng, V. Pardo, M. R. Norman, and J. F. Mitchell, Nat. Phys. 13, 864 (2017).

[27] G. A. Pan, D. F. Segedin, H. LaBollita, Q. Song, E. M. Nica, B. H. Goodge, A. T. Pierce, S. Doyle, S. Novakov, D. C. 
Carrizales, A. T. N'Diaye, P. Shafer, H. Paik, J. T. Heron, J. A. Mason, A. Yacoby, L. F. Kourkoutis, O. Erten, C. M. Brooks, A. S. Botana, and J. A. Mundy, arXiv:2109.09726 (2021).

[28] M. Wu, E. Benckiser, M. W. Haverkort, A. Frano, Y. Lu, U. Nwankwo, S. Brück, P. Audehm, E. Goering, S. Macke, V. Hinkov, P. Wochner, G. Christiani, S. Heinze, G. Logvenov, H. U. Habermeier, and B. Keimer, Phys. Rev. B 88, 125124 (2013).

[29] D. Di Castro, M. Salvato, A. Tebano, D. Innocenti, C. Aruta, W. Prellier, O. I. Lebedev, I. Ottaviani, N. B. Brookes, M. Minola, M. Moretti Sala, C. Mazzoli, P. G. Medaglia, G. Ghiringhelli, L. Braicovich, M. Cirillo, and G. Balestrino, Phys. Rev. B 86, 134524 (2012).

[30] S. Macke, J. E. Hamann-Borrero, R. J. Green, B. Keimer, G. A. Sawatzky, and M. W. Haverkort, Phys. Rev. Lett. 117, 115501 (2016).

[31] J. Stöhr and H. C. Siegmann, Magnetism: From Fundamentals to Nanoscale Dynamics (Springer, Berlin, 2006).

[32] R. J. Green, M. W. Haverkort, and G. A. Sawatzky, Phys. Rev. B 94, 195127 (2016).

[33] M. Jiang, M. Berciu, and G. A. Sawatzky, Phys. Rev. Lett. 124, 207004 (2020).

[34] C. T. Chen, L. H. Tjeng, J. Kwo, H. L. Kao, P. Rudolf, F. Sette, and R. M. Fleming, Phys. Rev. Lett. 68, 2543 (1992).

[35] R. I. Eglitis, J. Purans, and R. Jia, Crystals 11, 455 (2021).

[36] R. I. Eglitis, Appl. Surf. Sci. 358, 556 (2015).

[37] G. Kresse and J. Hafner, Phys. Rev. B 47, 558 (1993).

[38] J. P. Perdew, K. Burke, and M. Ernzerhof, Phys. Rev. Lett. 77, 3865 (1996).

[39] K. Koepernik and H. Eschrig, Phys. Rev. B 59, 1743 (1999).

[40] I. Opahle, K. Koepernik, and H. Eschrig, Phys. Rev. B 60, 14035 (1999).
[41] A. M. Oleś, Phys. Rev. B 28, 327 (1983).

[42] A. Georges, L. de Medici, and J. Mravlje, Annu. Rev. Condens. Matter Phys. 4, 137 (2013).

[43] L. Si, W. Xiao, J. Kaufmann, J. M. Tomczak, Y. Lu, Z. Zhong, and K. Held, Phys. Rev. Lett. 124, 166402 (2020).

[44] M. Wallerberger, A. Hausoel, P. Gunacker, A. Kowalski, N. Parragh, F. Goth, K. Held, and G. Sangiovanni, Comput. Phys. Commun. 235, 388 (2009).

[45] K. Held, Adv. Phys. 56, 829 (2007).

[46] Maxent: https://github.com/CQMP/Maxent.

[47] D. Toloui-Mantadakis, Ph.D. thesis, University of Stuttgart (2019).

[48] H. W. A. M. Rompa, M. F. H. Schuurmans, and F. Williams, Phys. Rev. Lett. 52, 675 (1984).

[49] N. Parragh, G. Sangiovanni, P. Hansmann, S. Hummel, K. Held, and A. Toschi, Phys. Rev. B 88, 195116 (2013).

[50] We stress the many-body nature of this effect, which is reflected in the dynamic nature of $\Sigma$ and the qualitative difference of orbital shifts at small (around $\varepsilon_{F}$ ) and large frequencies (corresponding to a static mean-field Hartree limit of the self-energy).

[51] L. de' Medici, J. Mravlje, and A. Georges, Phys. Rev. Lett. 107, 256401 (2011).

[52] L. Lechner, J. Biskupek, and U. Kaiser, Microsc. Microanal. 18, 379 (2012).

[53] C. L. Jia, M. Lentzen, and K. Urban, Science 299, 870 (2003).

[54] Q. Zhang, L. Zhang, C. Jin, Y. Wang, and F. Lin, Ultramicroscopy 202, 114 (2019).

[55] H. Y. Qi, M. K. Kinyanjui, J. Biskupek, D. Geiger, E. Benckiser, H.-U. Habermeier, B. Keimer, and U. Kaiser, J. Mater. Sci. 50, 5300 (2015). 